\title{
Massive MU-MIMO Downlink TDD Systems with Linear Precoding and Downlink Pilots
}

\author{
Hien Quoc Ngo*, Erik G. Larsson*, and Thomas L. Marzetta ${ }^{\dagger}$ \\ ${ }^{*}$ Department of Electrical Engineering (ISY) \\ Linköping University, 58183 Linköping, Sweden \\ ${ }^{\dagger}$ Bell Laboratories, Alcatel-Lucent \\ Murray Hill, NJ 07974, USA
}

\begin{abstract}
We consider a massive MU-MIMO downlink timedivision duplex system where a base station (BS) equipped with many antennas serves several single-antenna users in the same time-frequency resource. We assume that the BS uses linear precoding for the transmission. To reliably decode the signals transmitted from the BS, each user should have an estimate of its channel. In this work, we consider an efficient channel estimation scheme to acquire CSI at each user, called beamforming training scheme. With the beamforming training scheme, the BS precodes the pilot sequences and forwards to all users. Then, based on the received pilots, each user uses minimum mean-square error channel estimation to estimate the effective channel gains. The channel estimation overhead of this scheme does not depend on the number of BS antennas, and is only proportional to the number of users. We then derive a lower bound on the capacity for maximum-ratio transmission and zero-forcing precoding techniques which enables us to evaluate the spectral efficiency taking into account the spectral efficiency loss associated with the transmission of the downlink pilots. Comparing with previous work where each user uses only the statistical channel properties to decode the transmitted signals, we see that the proposed beamforming training scheme is preferable for moderate and low-mobility environments.
\end{abstract}

\section{INTRODUCTION}

Recently, massive (or very large) multiuser multiple-input multiple-output (MU-MIMO) systems have attracted a lot of attention from both academia and industry [1]-[4]. Massive MU-MIMO is a system where a base station (BS) equipped with many antennas simultaneously serves several users in the same frequency band. Owing to the large number of degreesof-freedom available for each user, massive MU-MIMO can provide a very high data rate and communication reliability with simple linear processing such as maximum-ratio combining (MRC) or zero-forcing (ZF) on the uplink and maximumratio transmission (MRT) or $\mathrm{ZF}$ on the downlink. At the same time, the radiated energy efficiency can be significantly improved [5]. Therefore, massive MU-MIMO is considered as a promising technology for next generations of cellular systems. In order to use the advantages that massive MUMIMO can offer, accurate channel state information (CSI) is required at the $\mathrm{BS}$ and/or the users.

The work of H. Q. Ngo and E. G. Larsson was supported in part by the Swedish Research Council (VR), the Swedish Foundation for Strategic Research (SSF), and ELLIIT.
In small MU-MIMO systems where the number of BS antennas is relatively small, typically, the BS can acquire an estimate of CSI via feedback in frequency-division duplex (FDD) operation [6]. More precisely, each user estimates the channels based on the downlink training, and then it feeds back its channel estimates to the BS through the reverse link. However, in massive MU-MIMO systems, the number of BS antennas is very large and channel estimation becomes challenging in FDD since the number of downlink resources needed for pilots will be proportional to the number of BS antennas. Also, the required bandwidth for CSI feedback becomes very large. By contrast, in time-division duplex (TDD) systems, owing to the channel reciprocity, the BS can obtain CSI in open-loop directly from the uplink training. The pilot transmission overhead is thus proportional to the number of users which is typically much smaller than the number of BS antennas. Therefore, CSI acquisition at the BS via open-loop training under TDD operation is preferable in massive MU-MIMO systems [1]-[3], [7], [8]. With this CSI acquisition, in the uplink, the signals transmitted from the users can be decoded by using these channel estimates. In the downlink, the BS can use the channel estimates to precode the transmit signals. However, the channel estimates are only available at the BS. The user also should have an estimate of the channel in order to reliably decode the transmitted signals in the downlink. To acquire CSI at the users, a simple scheme is that the BS sends the pilots to the users. Then, each user will estimate the channel based on the received pilots. This is very inefficient since the channel estimation overhead will be proportional to the number of BS antennas. Therefore, the majority of the research on these systems has assumed that the users do not have knowledge of the CSI. More precisely, the signal is detected at each user by only using the statistical properties of the channels [7]-[9]. Some work assumed that the users have perfect CSI [10]. To the authors' best knowledge, it has not been previously considered how to efficiently acquire CSI at each user in the massive MU-MIMO downlink.

In this paper, we propose a beamforming training scheme to acquire estimates of the CSI at each user. With this scheme, instead of forwarding a long pilot sequence (whose length is proportional to the number of BS antennas), the BS just beam- 
forms a short pilot sequence so that each user can estimate the effective channel gain (the combination of the precoding vector and the channel gain). The channel estimation overhead of this scheme is only proportional to the number of users. To evaluate the performance of the proposed beamforming training scheme, we derive a lower bound on the capacity of two specific linear precoding techniques, namely MRT and ZF. Numerical results show that the beamforming training scheme works very well in moderate and low-mobility environments.

Notation: We use upper (lower) bold letters to denote matrices (vectors). The superscripts $T, *$, and $H$ stand for the transpose, conjugate, and conjugate-transpose, respectively. $\operatorname{tr}(\boldsymbol{A})$ denotes the trace of a matrix $\boldsymbol{A}$, and $\boldsymbol{I}_{n}$ is the $n \times n$ identity matrix. The expectation operator and the Euclidean norm are denoted by $\mathbb{E}\{\cdot\}$ and $\|\cdot\|$, respectively. Finally, we use $z \sim \mathcal{C N}(0, \boldsymbol{\Sigma})$ to denote a circularly symmetric complex Gaussian vector $z$ with zero mean and covariance matrix $\Sigma$.

\section{System Model And BeAmforming TRAining}

We consider the downlink transmission in a MU-MIMO system where a BS equipped with $M$ antennas serves $K$ single-antenna users in the same time-frequency resource, see Fig. 1. Here, we assume that $M \gg K$. We further assume that the BS uses linear precoding techniques to process the signal before transmitting to all users. This requires knowledge of CSI at the BS. We assume TDD operation so that the channels on the uplink and downlink are equal. The estimates of CSI are obtained from uplink training.

\section{A. Uplink Training}

Let $\tau_{\mathrm{u}}$ be the number of symbols per coherence interval used entirely for uplink pilots. All users simultaneously transmit pilot sequences of length $\tau_{\mathrm{u}}$ symbols. The pilot sequences of $K$ users are pairwisely orthogonal. Therefore, it is required that $\tau_{\mathrm{u}} \geq K$.

Denote by $H \in \mathbb{C}^{M \times K}$ the channel matrix between the BS and the $K$ users. We assume that elements of $\boldsymbol{H}$ are i.i.d. Gaussian distributed with zero mean and unit variance. Here, for the simplicity, we neglect the effects of large-scale fading. Then, the minimum mean-square error (MMSE) estimate of $\boldsymbol{H}$ is given by [11]

$$
\hat{H}=\frac{\tau_{\mathrm{u}} p_{\mathrm{u}}}{\tau_{\mathrm{u}} p_{\mathrm{u}}+1} \boldsymbol{H}+\frac{\sqrt{\tau_{\mathrm{u}} p_{\mathrm{u}}}}{\tau_{\mathrm{u}} p_{\mathrm{u}}+1} \boldsymbol{N}_{\mathrm{u}}
$$

where $N_{\mathrm{u}}$ is a Gaussian matrix with i.i.d. $\mathcal{C N}(0,1)$ entries, and $p_{\mathrm{u}}$ denotes the average transmit power of each uplink pilot symbol. The channel matrix $\boldsymbol{H}$ can be decomposed as

$$
\boldsymbol{H}=\hat{\boldsymbol{H}}+\mathcal{E}
$$

where $\mathcal{E}$ is the channel estimation error. Since we use MMSE channel estimation, $\hat{\boldsymbol{H}}$ and $\mathcal{E}$ are independent [11]. Furthermore, $\hat{\boldsymbol{H}}$ has i.i.d. $\mathcal{C N}\left(0, \frac{\tau_{\mathrm{u}} p_{\mathrm{u}}}{\tau_{\mathrm{u}} p_{\mathrm{u}}+1}\right)$ elements, and $\mathcal{E}$ has i.i.d. $\mathcal{C N}\left(0, \frac{1}{\tau_{\mathrm{u}} p_{\mathrm{u}}+1}\right)$ elements.

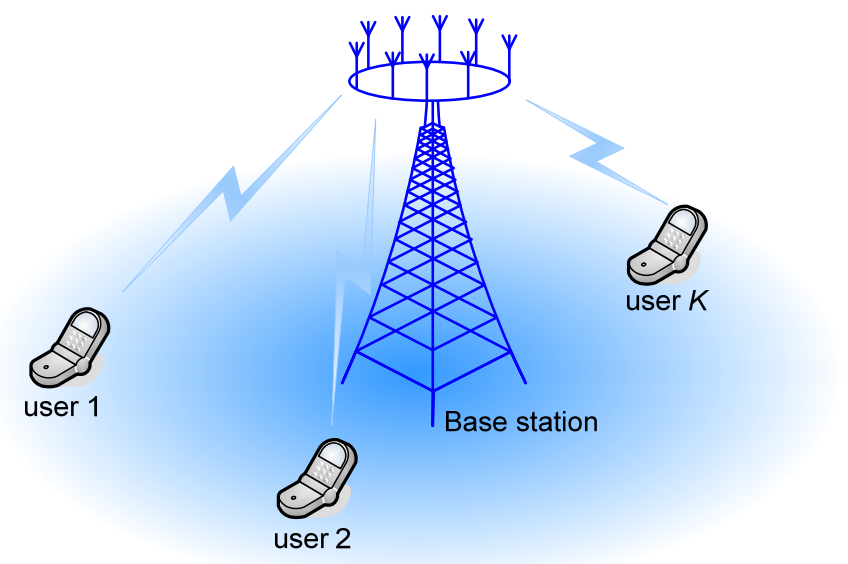

Fig. 1. Massive MU-MIMO downlink system model.

\section{B. Downlink Transmission}

Let $s_{k}$ be the symbol to be transmitted to the $k$ th user, with $\mathbb{E}\left\{\left|s_{k}\right|^{2}\right\}=1$. The BS uses the channel estimate $\hat{\boldsymbol{H}}$ to linearly precode the symbols, and then it transmits the precoded signal vector to all users. Let $W \in \mathbb{C}^{M \times K}$ be the linear precoding matrix which is a function of the channel estimate $\hat{\boldsymbol{H}}$. Then, the $M \times 1$ transmit signal vector is given by

$$
\boldsymbol{x}=\sqrt{p_{\mathrm{d}} W \boldsymbol{s}}
$$

where $s \triangleq\left[\begin{array}{llll}s_{1} & s_{2} & \ldots & s_{K}\end{array}\right]^{T}$, and $p_{\mathrm{d}}$ is the average transmit power at the BS. To satisfy the power constraint at the BS, $\boldsymbol{W}$ is chosen such as $\mathbb{E}\left\{\|\boldsymbol{x}\|^{2}\right\}=p_{\mathrm{d}}$, or equivalently $\mathbb{E}\left\{\operatorname{tr}\left(\boldsymbol{W} W^{H}\right)\right\}=1$.

The vector of samples collectively received at the $K$ users is given by

$$
\boldsymbol{y}=\boldsymbol{H}^{T} \boldsymbol{x}+\boldsymbol{n}=\sqrt{p_{\mathrm{d}}} \boldsymbol{H}^{T} \boldsymbol{W} \boldsymbol{s}+\boldsymbol{n}
$$

where $n$ is a vector whose $k$ th element, $n_{k}$, is the additive noise at the $k$ th user. We assume that $n_{k} \sim \mathcal{C N}(0,1)$. Define $a_{k i} \triangleq \boldsymbol{h}_{k}^{T} \boldsymbol{w}_{i}$, where $\boldsymbol{h}_{i}$ and $\boldsymbol{w}_{i}$ are the $i$ th columns of $\boldsymbol{H}$ and $W$, respectively. Then, the received signal at the $k$ th user can be written as

$$
y_{k}=\sqrt{p_{\mathrm{d}}} a_{k k} s_{k}+\sqrt{p_{\mathrm{d}}} \sum_{i \neq k}^{K} a_{k i} s_{i}+n_{k} .
$$

Remark 1: Each user should have CSI to coherently detect the transmitted signals. A simple way to acquire CSI is to use downlink pilots. The channel estimate overhead will be proportional to $M$. In massive MIMO, $M$ is large, so it is inefficient to estimate the full channel matrix $\boldsymbol{H}$ at each user using downlink pilots. This is the reason for why most of previous studies assumed that the users have only knowledge of the statistical properties of the channels [8], [9]. More precisely, in [8], [9], the authors use $\mathbb{E}\left\{a_{k k}\right\}$ to detect the transmitted signals. With very large $M, a_{k k}$ becomes nearly deterministic. In this case, using $\mathbb{E}\left\{a_{k k}\right\}$ for the signal detection is good enough. However, for moderately large $M$, the users should 
have CSI in order to reliably decode the transmitted signals. We observe from (5) that to detect $s_{k}$, user $k$ does not need the knowledge of $H$ (which has a dimension of $M \times K$ ). Instead, user $k$ needs only to know $a_{k k}$ which is a scalar value. Therefore, to acquire $a_{k k}$ at each user, we can spend a small amount of the coherence interval on downlink training. In the next section, we will provide more detail about this proposed downlink beamforming training scheme to estimate $a_{k k}$. With this scheme, the channel estimation overhead is proportional to the number of users $K$.

\section{Beamforming Training Scheme}

The BS beamforms the pilots. Then, the $k$ th user will estimate $a_{k i}$ by using the received pilots. Let $S_{\mathrm{p}} \in \mathbb{C}^{K \times \tau_{d}}$ be the pilot matrix, where $\tau_{\mathrm{d}}$ is the duration (in symbols) of the downlink training. The pilot matrix is given by

$$
S_{\mathrm{p}}=\sqrt{\tau_{\mathrm{d}} p_{\mathrm{d}}} \boldsymbol{\Phi} .
$$

We assume that the rows of $\Phi$ are pairwisely orthonormal, i.e., $\boldsymbol{\Phi} \boldsymbol{\Phi}^{H}=I_{K}$. This requires that $\tau_{d} \geq K$.

The BS beamforms the pilot sequence using the precoding matrix $W$. More precisely, the transmitted pilot matrix is $\boldsymbol{W} \boldsymbol{S}_{\mathrm{p}}$. Then, the $K \times \tau_{\mathrm{d}}$ received pilot matrix at the $K$ users is given by

$$
\boldsymbol{Y}_{\mathrm{p}}^{T}=\sqrt{\tau_{\mathrm{d}} p_{\mathrm{d}}} \boldsymbol{H}^{T} \boldsymbol{W} \boldsymbol{\Phi}+\boldsymbol{N}_{\mathrm{p}}^{T}
$$

where $N_{\mathrm{p}}$ is the AWGN matrix whose elements are i.i.d. $\mathcal{C N}(0,1)$. The received pilot matrix $\boldsymbol{Y}_{\mathrm{p}}^{T}$ can be represented by $\boldsymbol{Y}_{\mathrm{p}}^{T} \boldsymbol{\Phi}^{H}$ and $\boldsymbol{Y}_{\mathrm{p}}^{T} \boldsymbol{\Phi}_{\perp}^{H}$, where $\boldsymbol{\Phi}_{\perp}^{H}$ is the orthogonal complement of $\boldsymbol{\Phi}^{H}$, i.e., $\boldsymbol{\Phi}_{\perp}^{H}=\boldsymbol{I}_{\tau_{\mathrm{d}}}-\boldsymbol{\Phi}^{H} \boldsymbol{\Phi}$. We can see that $\boldsymbol{Y}_{\mathrm{p}}^{T} \boldsymbol{\Phi}_{\perp}^{H}$ only includes noise which is independent of $\boldsymbol{Y}_{\mathrm{p}}^{T} \boldsymbol{\Phi}^{H}$. Thus, it is sufficient to use $Y_{\mathrm{p}}^{T} \boldsymbol{\Phi}^{H}$ for the channel estimation. Let

$$
\tilde{\boldsymbol{Y}}_{\mathrm{p}}^{T} \triangleq \boldsymbol{Y}_{\mathrm{p}}^{T} \boldsymbol{\Phi}^{H}=\sqrt{\tau_{\mathrm{d}} p_{\mathrm{d}}} \boldsymbol{H}^{T} \boldsymbol{W}+\tilde{\boldsymbol{N}}_{\mathrm{p}}^{T}
$$

where $\tilde{\boldsymbol{N}}_{\mathrm{p}}^{T} \triangleq \boldsymbol{N}_{\mathrm{p}}^{T} \boldsymbol{\Phi}^{H}$ has i.i.d. $\mathcal{C} \mathcal{N}(0,1)$ elements. From $[8]$, the $1 \times K$ received pilot vector at user $k$ is given by

$$
\tilde{\boldsymbol{y}}_{\mathrm{p}, k}^{T}=\sqrt{\tau_{\mathrm{d}} p_{\mathrm{d}}} \boldsymbol{h}_{k}^{T} \boldsymbol{W}+\tilde{\boldsymbol{n}}_{\mathrm{p}, k}^{T}=\sqrt{\tau_{\mathrm{d}} p_{\mathrm{d}}} \boldsymbol{a}_{k}^{T}+\tilde{\boldsymbol{n}}_{\mathrm{p}, k}^{T}
$$

where $\boldsymbol{a}_{k} \triangleq\left[\begin{array}{llll}a_{k 1} & a_{k 2} & \ldots & a_{k K}\end{array}\right]^{T}$, and $\tilde{\boldsymbol{y}}_{\mathrm{p}, k}$ and $\tilde{\boldsymbol{n}}_{\mathrm{p}, k}$ are the $k$ th columns of $\tilde{\boldsymbol{Y}}_{\mathrm{p}}$ and $\tilde{\boldsymbol{N}}_{\mathrm{p}}$, respectively.

From the received pilot $\tilde{\boldsymbol{y}}_{\mathrm{p}, k}^{T}$, user $k$ estimates $\boldsymbol{a}_{k}$. Depending on the precoding matrix $\boldsymbol{W}$, the elements of $\boldsymbol{a}_{k}$ can be correlated and hence, they should be jointly estimated. However, here, for the simplicity of the analysis, we estimate $a_{k 1}, \ldots, a_{k K}$ independently, i.e., we use the $i$ th element of $\tilde{\boldsymbol{y}}_{p, k}$ to estimate $a_{k i}$. In Section V we show that estimating the elements of $a_{k}$ jointly will not improve the system performance much compared to the case where the elements of $\boldsymbol{a}_{k}$ are estimated independently. The MMSE channel estimate of $a_{k i}$ is given by [11]

$\hat{a}_{k i}=\mathbb{E}\left\{a_{k i}\right\}+\frac{\sqrt{\tau_{\mathrm{d}} p_{\mathrm{d}} \operatorname{Var}\left(a_{k i}\right)}}{\tau_{\mathrm{d}} p_{\mathrm{d}} \operatorname{Var}\left(a_{k i}\right)+1}\left(\tilde{y}_{\mathrm{p}, k i}-\sqrt{\tau_{\mathrm{d}} p_{\mathrm{d}}} \mathbb{E}\left\{a_{k i}\right\}\right)$ where $\operatorname{Var}\left(a_{k i}\right) \triangleq \mathbb{E}\left\{\left|a_{k i}-\mathbb{E}\left\{a_{k i}\right\}\right|^{2}\right\}$, and $\tilde{y}_{\mathrm{p}, k i}$ is the $i$ th element of $\tilde{\boldsymbol{y}}_{\mathrm{p}, k}$. Let $\epsilon_{k i}$ be the channel estimation error. Then, the effective channel $a_{k i}$ can be decomposed as

$$
a_{k i}=\hat{a}_{k i}+\epsilon_{k i} \text {. }
$$

Note that, since we use MMSE estimation, the estimate $\hat{a}_{k i}$ and the estimation error $\epsilon_{k i}$ are uncorrelated.

\section{Achievable Downlink Rate}

In this section, we derive a lower bound on the achievable downlink rate for MRT and ZF precoding techniques, using the proposed beamforming training scheme. To obtain these achievable rates, we use the techniques of [12].

User $k$ uses the channel estimate $\hat{a}_{k}$ in 10 to detect the transmitted signal $s_{k}$. Therefore, the achievable downlink rate of the transmission from the BS to the $k$ th user is the mutual information between the unknown transmitted signal $s_{k}$ and the observed received signal $y_{k}$ given by (5) and the known channel estimate $\hat{\boldsymbol{a}}_{k}=\left[\begin{array}{lll}\hat{a}_{k 1} & \ldots & \hat{a}_{k K}\end{array}\right]^{T}$, i.e., $I\left(s_{k} ; y_{k}, \hat{\boldsymbol{a}}_{k}\right)$.

Following a similar methodology as in [12, Appendix A], we obtain a lower bound on the achievable rate of the transmission from the BS to the $k$ th user as:

$$
R_{k}=\mathbb{E}\left\{\log _{2}\left(1+\frac{p_{\mathrm{d}}\left|\hat{a}_{k k}\right|^{2}}{p_{\mathrm{d}} \sum_{i=1}^{K} \mathbb{E}\left\{\left|\epsilon_{k i}\right|^{2}\right\}+p_{\mathrm{d}} \sum_{i \neq k}^{K}\left|\hat{a}_{k i}\right|^{2}+1}\right)\right\} .
$$

We next simplify the capacity lower bound given by 12 for two specific linear precoding techniques at the BS, namely, MRT and ZF.

\section{A. Maximum-Ratio Transmission}

With MRT, the precoding matrix $W$ is given by

$$
\boldsymbol{W}=\alpha_{\mathrm{MRT}} \hat{\boldsymbol{H}}^{*}
$$

where $\alpha_{\mathrm{MRT}}$ is a normalization constant chosen to satisfy the transmit power constraint at the BS, i.e., $\mathbb{E}\left\{\operatorname{tr}\left(W W^{H}\right)\right\}=$ 1. Hence,

$$
\alpha_{\mathrm{MRT}}=\sqrt{\frac{1}{\mathbb{E}\left\{\operatorname{tr}\left(\hat{\boldsymbol{H}}^{*} \hat{\boldsymbol{H}}^{T}\right)\right\}}}=\sqrt{\frac{\tau_{\mathrm{u}} p_{\mathrm{u}}+1}{M K \tau_{\mathrm{u}} p_{\mathrm{u}}}} .
$$

Proposition 1: With MRT, the lower bound on the achievable rate given by 12 becomes

$$
R_{k}=\mathbb{E}\left\{\log _{2}\left(1+\frac{p_{\mathrm{d}}\left|\hat{a}_{k k}\right|^{2}}{\frac{K p_{\mathrm{d}}}{\tau_{\mathrm{d}} p_{\mathrm{d}}+K}+p_{\mathrm{d}} \sum_{i \neq k}^{K}\left|\hat{a}_{k i}\right|^{2}+1}\right)\right\}
$$

where

$$
\hat{a}_{k i}=\frac{\sqrt{\tau_{\mathrm{d}} p_{\mathrm{d}}}}{\tau_{\mathrm{d}} p_{\mathrm{d}}+K} \tilde{\boldsymbol{y}}_{p, k i}+\frac{K}{\tau_{\mathrm{d}} p_{\mathrm{d}}+K} \sqrt{\frac{\tau_{\mathrm{u}} p_{\mathrm{u}} M}{K\left(\tau_{\mathrm{u}} p_{\mathrm{u}}+1\right)}} \delta_{k i}
$$

where $\delta_{k i}=1$ when $i=k$ and 0 otherwise.

Proof: See Appendix A 


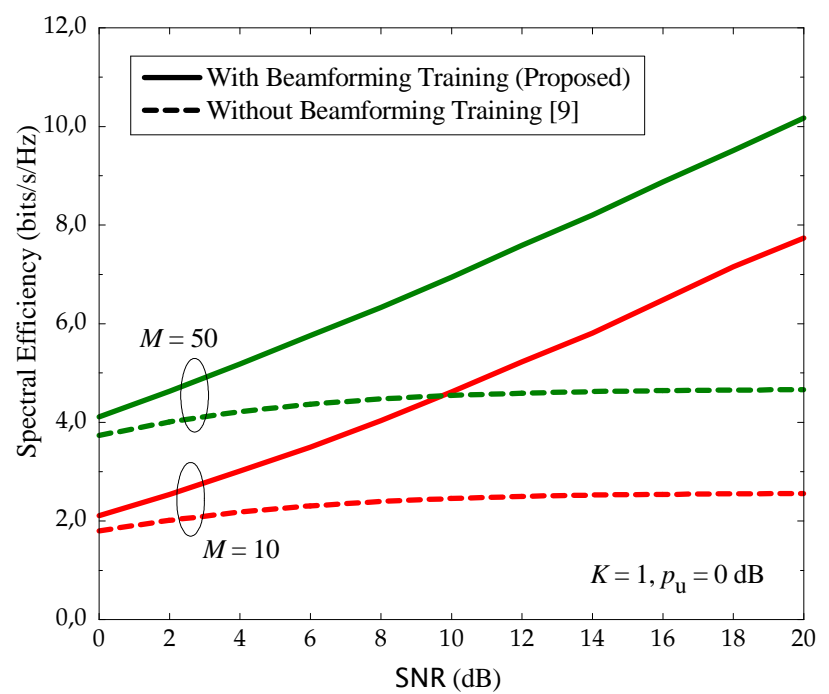

Fig. 2. Spectral efficiency versus SNR for a single-user setup $(K=1$, $p_{\mathrm{u}}=0 \mathrm{~dB}$, and $T=200$ ).

\section{B. Zero-Forcing}

With $\mathrm{ZF}$, the precoding matrix is

$$
W=\alpha_{\mathrm{ZF}} \hat{\boldsymbol{H}}^{*}\left(\hat{\boldsymbol{H}}^{T} \hat{\boldsymbol{H}}^{*}\right)^{-1}
$$

where the normalization constant $\alpha_{\text {ZF }}$ is chosen to satisfy the power constraint $\mathbb{E}\left\{\operatorname{tr}\left(\boldsymbol{W} \boldsymbol{W}^{H}\right)\right\}=1$, i.e.,

$$
\alpha_{\mathrm{ZF}}=\sqrt{\frac{(M-K) \tau_{\mathrm{u}} p_{\mathrm{u}}}{K\left(\tau_{\mathrm{u}} p_{\mathrm{u}}+1\right)}} .
$$

Proposition 2: With ZF, the lower bound on the achievable rate given by (12) becomes

$$
R_{k}=\mathbb{E}\left\{\log _{2}\left(1+\frac{p_{\mathrm{d}}\left|\hat{a}_{k k}\right|^{2}}{\frac{K p_{\mathrm{d}}}{\tau_{\mathrm{d}} p_{\mathrm{d}}+K\left(\tau_{\mathrm{u}} p_{\mathrm{u}}+1\right)}+p_{\mathrm{d}} \sum_{i \neq k}^{K}\left|\hat{a}_{k i}\right|^{2}+1}\right)\right\}
$$

where

$$
\begin{aligned}
\hat{a}_{k i} & =\frac{\sqrt{\tau_{\mathrm{d}} p_{\mathrm{d}}}}{\tau_{\mathrm{d}} p_{\mathrm{d}}+K\left(\tau_{\mathrm{u}} p_{\mathrm{u}}+1\right)} \tilde{\boldsymbol{y}}_{p, k i} \\
& +\frac{\sqrt{K(M-K) \tau_{\mathrm{u}} p_{\mathrm{u}}\left(\tau_{\mathrm{u}} p_{\mathrm{u}}+1\right)}}{\tau_{\mathrm{d}} p_{\mathrm{d}}+K\left(\tau_{\mathrm{u}} p_{\mathrm{u}}+1\right)} \delta_{k i} .
\end{aligned}
$$

Proof: See Appendix B

\section{Numerical Results}

In this section, we illustrate the spectral efficiency performance of the beamforming training scheme. The spectral efficiency is defined as the sum-rate (in bits) per channel use. Let $T$ be the length of the coherence interval (in symbols). During each coherence interval, we spend $\tau_{\mathrm{u}}$ symbols for uplink training and $\tau_{\mathrm{d}}$ symbols for beamforming training. Therefore, the spectral efficiency is given by

$$
\mathcal{S}_{\mathrm{TB}}=\frac{T-\tau_{\mathrm{u}}-\tau_{\mathrm{d}}}{T} \sum_{k=1}^{K} R_{k}
$$

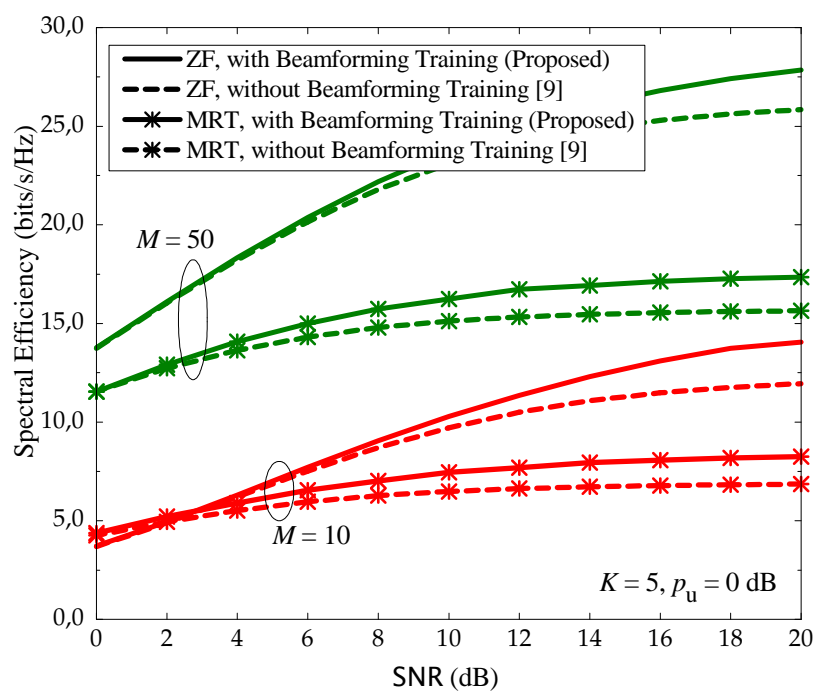

Fig. 3. Spectral efficiency versus SNR for a multiuser setup $\left(K=5, p_{\mathrm{u}}=0\right.$ $\mathrm{dB}$, and $T=200$ ).

where $R_{k}$ is given by (15) for MRT, and (19) for ZF.

For comparison, we also consider the spectral efficiency for the case that there is no beamforming training and that $\mathbb{E}\left\{a_{k k}\right\}$ is used instead of $a_{k k}$ for the detection [9]. The spectral efficiency for this case is given by [9]

$$
\mathcal{S}_{0}=\left\{\begin{array}{l}
\frac{T-\tau_{\mathrm{u}}}{T} K \log _{2}\left(1+\frac{M}{K} \frac{\tau_{\mathrm{u}} p_{\mathrm{u}} p_{\mathrm{d}}}{\left(p_{\mathrm{d}}+1\right)\left(\tau_{\mathrm{u}} p_{\mathrm{u}}+1\right)}\right), \text { for MRT } \\
\frac{T-\tau_{\mathrm{u}}}{T} K \log _{2}\left(1+\frac{M-K}{K} \frac{\tau_{\mathrm{u}} p_{\mathrm{u}} p_{\mathrm{d}}}{\tau_{\mathrm{u}} p_{\mathrm{u}}+p_{\mathrm{d}}+1}\right), \text { for ZF }
\end{array}\right.
$$

In all examples, we choose $\tau_{\mathrm{u}}=\tau_{\mathrm{d}}=K$ and $p_{\mathrm{u}}=0 \mathrm{~dB}$. We define $\mathrm{SNR} \triangleq p_{\mathrm{d}}$.

We first consider a single-user setup $(K=1)$. When $K=1$, the performances MRT and ZF are the same. Fig. 2 shows the spectral efficiency versus SNR for different number of BS antennas $M=10$ and $M=50$, at $T=200$ (e.g. $1 \mathrm{~ms} \times 200 \mathrm{kHz}$ ). We can see that the beamforming training scheme outperforms the case without beamforming training. The performance gap increases significantly when the SNR increases. The reason is that, when SNR (or the downlink power) increases, the channel estimate at each user is more accurate and hence, the advantage of the beamforming training scheme grows.

Next, we consider a multiuser setup. Here, we choose the number of users to be $K=5$. Fig. 3 shows the spectral efficiency versus SNR for the MRT and ZF precoders, at $M=10, M=50$, and $T=200$. Again, the beamforming training offers an improved performance. In addition, we can see that the beamforming training with MRT precoding is more efficient than the beamforming training with $\mathrm{ZF}$ precoding. This is due to the fact that, with $\mathrm{ZF}$, the randomness of the effective channel gain $a_{k k}$ at the $k$ th user is smaller than the one with MRT (with ZF, $a_{k k}$ becomes deterministic when the BS has perfect CSI) and hence, MRC has a higher advantage of using the channel estimate for the signal detection.

Furthermore, we consider the effect of the length of the coherence interval on the system performance of the beam- 


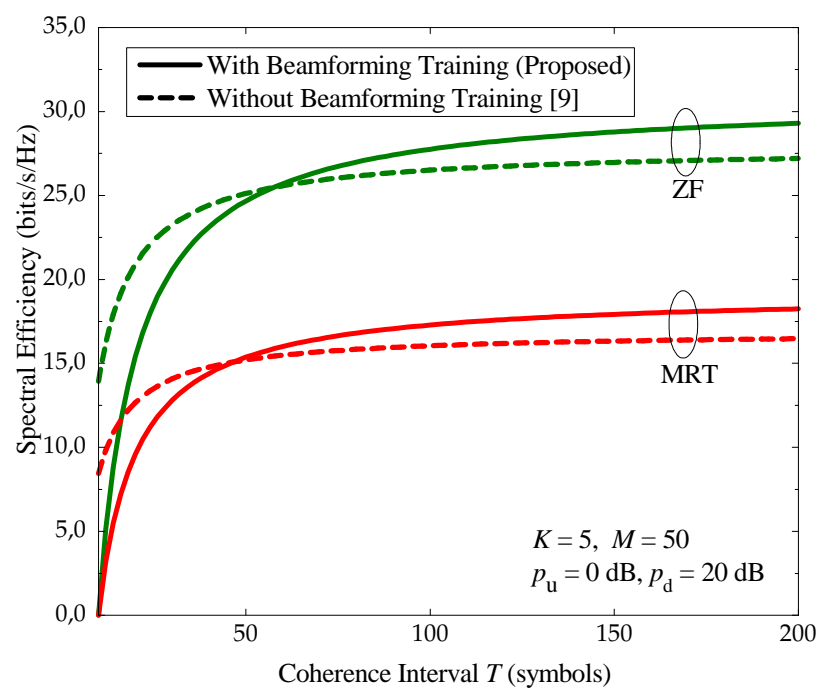

Fig. 4. Spectral efficiency versus coherence interval for MRT and ZF precoding $\left(M=50, K=5, p_{\mathrm{u}}=0 \mathrm{~dB}\right.$, and $\left.p_{\mathrm{d}}=20 \mathrm{~dB}\right)$.

forming training scheme. Fig. 4 4 shows the spectral efficiency versus the length of the coherence interval $T$ at $M=50$, $K=5$, and $p_{\mathrm{d}}=20 \mathrm{~dB}$. As expected, for short coherence intervals (in a high-mobility environment), the training duration is relatively large compared to the length of the coherence interval and hence, we should not use the beamforming training to estimate CSI at each user. At moderate and large $T$, the training duration is relatively small compared with the coherence interval. As a result, the beamforming training scheme is preferable.

Finally, we consider the spectral efficiency of our scheme but with a genie receiver, i.e., we assume that the $k$ th user can estimate perfectly $\boldsymbol{a}_{k}$ in the beamforming training phase. For this case, the spectral efficiency is given by

$$
\mathcal{S}_{\mathrm{G}}=\frac{T-\tau_{\mathrm{u}}-\tau_{\mathrm{d}}}{T} \sum_{k=1}^{K} \mathbb{E}\left\{\log _{2}\left(1+\frac{p_{\mathrm{d}}\left|a_{k k}\right|^{2}}{p_{\mathrm{d}} \sum_{i \neq k}^{K}\left|a_{k i}\right|^{2}+1}\right)\right\} \text {. }
$$

Figure 5 compares the spectral efficiency given by (12), where the $k$ th user estimates the elements of $\boldsymbol{a}_{k}$ independently, with the one obtained by (23), where we assume that there is a genie receiver at the $k$ th user. Here, we choose $K=5$ and $T=200$. We can see that performance gap between two cases is very small. This implies that estimating the elements of $\boldsymbol{a}_{k}$ independently is fairly reasonable.

\section{CONCLUSion AND Future Work}

In this paper, we proposed and analyzed a scheme to acquire CSI at each user in the downlink of a MU-MIMO system, called beamforming training scheme. With this scheme, the BS uses linear precoding techniques to process the pilot sequence before sending it to the users for the channel estimation. The channel estimation overhead of this beamforming training scheme is small and does not depend on the number of BS

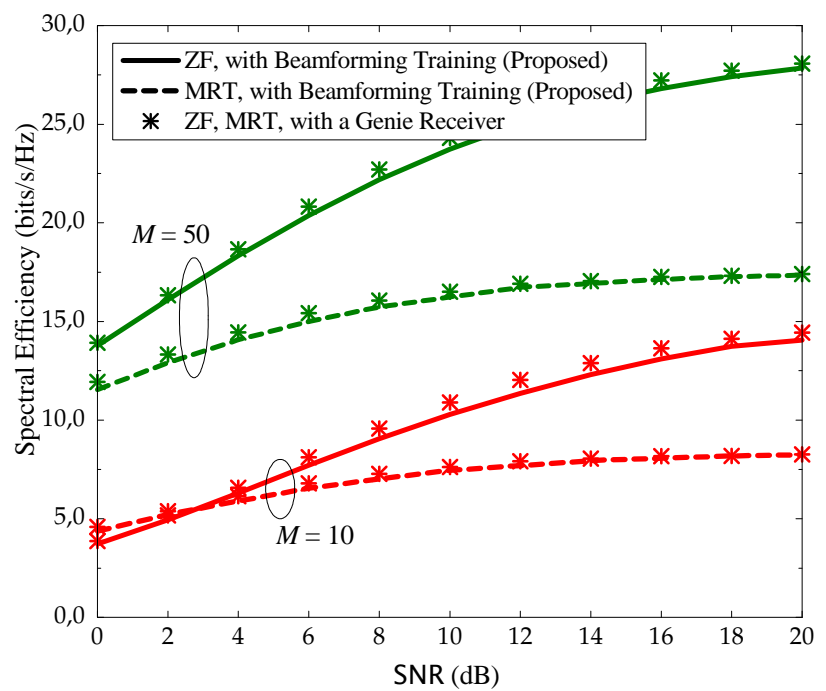

Fig. 5. Spectral efficiency versus SNR with a genie receiver $\left(K=5, p_{\mathrm{u}}=0\right.$ $\mathrm{dB}$, and $T=200)$.

antennas. Therefore, it is suitable and efficient for massive MU-MIMO systems. Furthermore, the down-link pilots will add robustness to the beamforming process which otherwise is dependent on the validity of the prior (Bayes) assumptions.

\section{APPENDIX}

\section{A. Proof of Proposition $\square$}

With MRT, we have that $a_{k i}=\alpha_{\text {MRT }} \boldsymbol{h}_{k}^{T} \hat{\boldsymbol{h}}_{i}^{*}$.

- Compute $\mathbb{E}\left\{a_{k i}\right\}$ :

From (2), we have

$$
\begin{aligned}
a_{k i} & =\alpha_{\mathrm{MRT}}\left(\hat{\boldsymbol{h}}_{k}^{T}+\boldsymbol{\varepsilon}_{k}^{T}\right) \hat{\boldsymbol{h}}_{i}^{*} \\
& =\alpha_{\mathrm{MRT}} \hat{h}_{k}^{T} \hat{\boldsymbol{h}}_{i}^{*}+\alpha_{\mathrm{MRT}} \varepsilon_{k}^{T} \hat{\boldsymbol{h}}_{i}^{*}
\end{aligned}
$$

where $\hat{\boldsymbol{h}}_{k}$ and $\boldsymbol{\varepsilon}_{k}$ are the $k$ th columns of $\hat{\boldsymbol{H}}$ and $\mathcal{E}$, respectively. Since $\hat{\varepsilon}_{k}$ and $\hat{h}_{i}^{*}$ are uncorrelated with all $i, k=1, \ldots, K$, we obtain

$$
\begin{aligned}
\mathbb{E}\left\{a_{k i}\right\} & =\alpha_{\text {MRT }} \mathbb{E}\left\{\hat{\boldsymbol{h}}_{k}^{T} \hat{\boldsymbol{h}}_{i}^{*}\right\} \\
& =\left\{\begin{array}{l}
0, \text { if } i \neq k \\
\sqrt{\frac{\tau_{\mathrm{u}} p_{\mathrm{u}} M}{K\left(\tau_{\mathrm{u}} p_{\mathrm{u}}+1\right)}}, \text { if } i=k
\end{array}\right.
\end{aligned}
$$

- Compute $\operatorname{Var}\left(a_{k i}\right)$ for $i \neq k$ :

From (24) and (25), we have

$$
\begin{aligned}
\operatorname{Var}\left(a_{k i}\right) & =\mathbb{E}\left\{\left|a_{k i}\right|^{2}\right\} \\
& \stackrel{(a)}{=} \mathbb{E}\left\{\left|\alpha_{\mathrm{MRT}} \hat{\boldsymbol{h}}_{k}^{T} \hat{\boldsymbol{h}}_{i}^{*}\right|^{2}\right\}+\mathbb{E}\left\{\left|\alpha_{\mathrm{MRT}} \varepsilon_{k}^{T} \hat{\boldsymbol{h}}_{i}^{*}\right|^{2}\right\} \\
& =\alpha_{\mathrm{MRT}}^{2}\left(\frac{\tau_{\mathrm{u}} p_{\mathrm{u}}}{\tau_{\mathrm{u}} p_{\mathrm{u}}+1}\right)^{2} M+\alpha_{\mathrm{MRT}}^{2} \frac{\tau_{\mathrm{u}} p_{\mathrm{u}} M}{\left(\tau_{\mathrm{u}} p_{\mathrm{u}}+1\right)^{2}} \\
& =1 / K
\end{aligned}
$$

where (a) is obtained by using the fact that $\hat{\boldsymbol{h}}_{k}^{T} \hat{\boldsymbol{h}}_{i}^{*}$ and $\boldsymbol{\varepsilon}_{k}^{T} \hat{\boldsymbol{h}}_{i}^{*}$ are uncorrelated. 
- Compute $\operatorname{Var}\left(a_{k k}\right)$ :

Similarly, we have

$$
\operatorname{Var}\left(a_{k k}\right)=\mathbb{E}\left\{\left|a_{k k}\right|^{2}\right\}-\left|\mathbb{E}\left\{a_{k k}\right\}\right|^{2} .
$$

From (24), we have

$$
\mathbb{E}\left\{\left|a_{k k}\right|^{2}\right\}=\alpha_{\mathrm{MRT}}^{2} \mathbb{E}\left\{\left\|\hat{\boldsymbol{h}}_{k}\right\|^{4}\right\}+\alpha_{\mathrm{MRT}}^{2} \mathbb{E}\left\{\left|\varepsilon_{k}^{T} \hat{\boldsymbol{h}}_{k}^{*}\right|^{2}\right\} .
$$

Using [13, Lemma 2.9], we obtain

$$
\begin{aligned}
\mathbb{E}\left\{\left|a_{k k}\right|^{2}\right\} & =\alpha_{\mathrm{MRT}}^{2}\left(\frac{\tau_{\mathrm{u}} p_{\mathrm{u}}}{\tau_{\mathrm{u}} p_{\mathrm{u}}+1}\right)^{2} M(M+1) \\
& +\alpha_{\mathrm{MRT}}^{2} \frac{\tau_{\mathrm{u}} p_{\mathrm{u}}}{\left(\tau_{\mathrm{u}} p_{\mathrm{u}}+1\right)^{2}} M
\end{aligned}
$$

Substituting (25) and (29) into (27), we obtain

$$
\operatorname{Var}\left(a_{k k}\right)=1 / K \text {. }
$$

Substituting (25), (26), and (30) into (10), we get (16).

- Compute $\mathbb{E}\left\{\left|\epsilon_{k i}\right|^{2}\right\}$ :

If $i \neq k$, from (9) and (16), we have

$$
\begin{aligned}
& \mathbb{E}\left\{\left|\epsilon_{k i}\right|^{2}\right\}=\mathbb{E}\left\{\left|a_{k i}-\hat{a}_{k i}\right|^{2}\right\} \\
& =\mathbb{E}\left\{\left|\frac{K}{\tau_{\mathrm{d}} p_{\mathrm{d}}+K} a_{k i}-\frac{\sqrt{\tau_{\mathrm{d}} p_{\mathrm{d}}}}{\tau_{\mathrm{d}} p_{\mathrm{d}}+K} \tilde{n}_{\mathrm{p}, k i}\right|^{2}\right\} \\
& =\left(\frac{K}{\tau_{\mathrm{d}} p_{\mathrm{d}}+K}\right)^{2} \mathbb{E}\left\{\left|a_{k i}\right|^{2}\right\}+\frac{\tau_{\mathrm{d}} p_{\mathrm{d}}}{\left(\tau_{\mathrm{d}} p_{\mathrm{d}}+K\right)^{2}}
\end{aligned}
$$

where $\tilde{n}_{\mathrm{p}, k i}$ is the $i$ th element of $\tilde{\boldsymbol{n}}_{\mathrm{p}, k}$. Using 26), we obtain

$$
\mathbb{E}\left\{\left|\epsilon_{k i}\right|^{2}\right\}=\frac{1}{\tau_{\mathrm{d}} p_{\mathrm{d}}+K} .
$$

Similarly, we obtain $\mathbb{E}\left\{\left|\epsilon_{k k}\right|^{2}\right\}=\frac{1}{\tau_{\mathrm{d}} p_{\mathrm{d}}+K}$. Therefore, we arrive at the result in Proposition 1

\section{B. Proof of Proposition 2}

With ZF, we have that $a_{k i}=\boldsymbol{h}_{k}^{T} \boldsymbol{w}_{i}$, where $\boldsymbol{w}_{i}$ is the $i$ th column of $\alpha_{\mathrm{ZF}} \hat{\boldsymbol{H}}^{*}\left(\hat{\boldsymbol{H}}^{T} \hat{\boldsymbol{H}}^{*}\right)^{-1}$. Since $\hat{\boldsymbol{H}}^{T} \boldsymbol{W}=\alpha_{\mathrm{ZF}} \boldsymbol{I}_{K}$, we have

$$
a_{k i}=\left(\hat{\boldsymbol{h}}_{k}^{T}+\boldsymbol{\varepsilon}_{k}^{T}\right) \boldsymbol{w}_{i}=\alpha_{\mathrm{ZF}} \delta_{k i}+\boldsymbol{\varepsilon}_{k}^{T} \boldsymbol{w}_{i} .
$$

Therefore,

$$
\mathbb{E}\left\{a_{k i}\right\}=\alpha_{\mathrm{ZF}} \delta_{k i} .
$$

- Compute $\operatorname{Var}\left(a_{k i}\right)$ :

From (33) and (34), we have

$$
\begin{aligned}
\operatorname{Var}\left(a_{k i}\right) & =\mathbb{E}\left\{\left|\boldsymbol{\varepsilon}_{k}^{T} \boldsymbol{w}_{i}\right|^{2}\right\}=\frac{1}{\tau_{\mathrm{u}} p_{\mathrm{u}}+1} \mathbb{E}\left\{\left\|\boldsymbol{w}_{i}\right\|^{2}\right\} \\
& =\frac{\alpha_{\mathrm{ZF}}^{2}}{\tau_{\mathrm{u}} p_{\mathrm{u}}+1} \mathbb{E}\left\{\left[\left(\hat{\boldsymbol{H}}^{T} \hat{\boldsymbol{H}}^{*}\right)^{-1}\right]_{i i}\right\} \\
& =\frac{\alpha_{\mathrm{ZF}}^{2}}{\tau_{\mathrm{u}} p_{\mathrm{u}}+1} \frac{1}{K} \mathbb{E}\left\{\operatorname{tr}\left[\left(\hat{\boldsymbol{H}}^{T} \hat{\boldsymbol{H}}^{*}\right)^{-1}\right]\right\} .
\end{aligned}
$$

Using [13, Lemma 2.10], we obtain

$$
\operatorname{Var}\left(a_{k i}\right)=\frac{1}{K\left(\tau_{\mathrm{u}} p_{\mathrm{u}}+1\right)} .
$$

Substituting (34) and (36) into (10), we get 20).

- Compute $\mathbb{E}\left\{\left|\epsilon_{k i}\right|^{2}\right\}$ :

If $i \neq k$, from (9) and (20), we have

$$
\begin{aligned}
& \mathbb{E}\left\{\left|\epsilon_{k i}\right|^{2}\right\}=\mathbb{E}\left\{\left|a_{k i}-\hat{a}_{k i}\right|^{2}\right\} \\
& =\mathbb{E}\left\{\left|\frac{K\left(\tau_{\mathrm{u}} p_{\mathrm{u}}+1\right) a_{k i}}{\tau_{\mathrm{d}} p_{\mathrm{d}}+K\left(\tau_{\mathrm{u}} p_{\mathrm{u}}+1\right)}-\frac{\sqrt{\tau_{\mathrm{d}} p_{\mathrm{d}}} \tilde{n}_{\mathrm{p}, k i}}{\tau_{\mathrm{d}} p_{\mathrm{d}}+K\left(\tau_{\mathrm{u}} p_{\mathrm{u}}+1\right)}\right|^{2}\right\} \\
& =\left(\frac{K\left(\tau_{\mathrm{u}} p_{\mathrm{u}}+1\right)}{\tau_{\mathrm{d}} p_{\mathrm{d}}+K\left(\tau_{\mathrm{u}} p_{\mathrm{u}}+1\right)}\right)^{2} \mathbb{E}\left\{\left|a_{k i}\right|^{2}\right\} \\
& +\frac{\tau_{\mathrm{d}} p_{\mathrm{d}}}{\left(\tau_{\mathrm{d}} p_{\mathrm{d}}+K\left(\tau_{\mathrm{u}} p_{\mathrm{u}}+1\right)\right)^{2}} \\
& =\frac{1}{\tau_{\mathrm{d}} p_{\mathrm{d}}+K\left(\tau_{\mathrm{u}} p_{\mathrm{u}}+1\right)}
\end{aligned}
$$

where the last equality is obtained by using (36). Similarly, we obtain $\mathbb{E}\left\{\left|\epsilon_{k k}\right|^{2}\right\}=\frac{1}{\tau_{\mathrm{d}} p_{\mathrm{d}}+K\left(\tau_{\mathrm{u}} p_{\mathrm{u}}+1\right)}$. Therefore, we arrive at the result in Proposition 2 .

\section{REFERENCES}

[1] T. L. Marzetta, "Noncooperative cellular wireless with unlimited numbers of base station antennas," IEEE Trans. Wireless Commun., vol. 9, no. 11 , pp. $3590-3600$, Nov. 2010.

[2] E. G. Larsson, F. Tufvesson, O. Edfors, and T. L. Marzetta, "Massive MIMO for next generation wireless systems," IEEE Commun. Mag., 2013, to appear. [Online]. Available: http://arxiv.org/abs/1304.6690

[3] Y.-H. Nam, B. L. Ng, K. Sayana, Y. Li, J. C. Zhang, Y. Kim, and J. Lee, "Full-dimension MIMO (FD-MIMO) for next generation cellular technology," IEEE Commun. Mag., vol. 51, no. 6, pp. 172-178, 2013.

[4] C. Shepard, H. Yu, N. Anand, L. E. Li, T. L. Marzetta, R. Yang, and L. Zhong, "Argos: Practical many-antenna base stations," in Proc. ACM Int. Conf. Mobile Computing and Networking (MobiCom), Istanbul, Turkey, Aug. 2012.

[5] H. Q. Ngo, E. G. Larsson, and T. L. Marzetta, "Energy and spectral efficiency of very large multiuser MIMO systems," IEEE Trans. Commun., vol. 61, no. 4, pp. 1436-1449, Apr. 2013.

[6] M. Kobayashi, N. Jindal, and G. Caire, "Training and feedback optimization for multiuser MIMO downlink," IEEE Trans. Commun., vol. 59, no. 8, pp. 2228-2240, Aug. 2011.

[7] J. Hoydis, S. ten Brink, and M. Debbah, "Massive MIMO in the UL/DL of cellular networks: How many antennas do we need?" IEEE J. Sel. Areas Commun., vol. 31, no. 2, pp. 160-171, Feb. 2013.

[8] J. Jose, A. Ashikhmin, T. L. Marzetta, and S. Vishwanath, "Pilot contamination and precoding in multi-cell TDD systems," IEEE Trans. Wireless Commun., vol. 10, no. 8, pp. 2640-2651, Aug. 2011.

[9] H. Yang and T. L. Marzetta, "Performance of conjugate and zeroforcing beamforming in large-scale antenna systems," IEEE J. Sel. Areas Commun., vol. 31, no. 2, pp. 172-179, Feb. 2013.

[10] E. Björnson, J. Hoydis, M. Kountouris, and M. Debbah, "Hardware impairments in large-scale MISO systems: Energy efficiency, estimation, and capacity limits," in Proc. Signal Processing and Optimization for Green Energy and Green Communications (DSP'13), Santorini, Greece, 2013.

[11] S. M. Kay, Fundamentals of Statistical Signal Processing: Estimation Theory. Englewood Cliffs, NJ: Prentice Hall, 1993.

[12] H. Q. Ngo, E. G. Larsson, and T. L. Marzetta, "The multicell multiuser MIMO uplink with very large antenna arrays and a finite-dimensional channel," IEEE Trans. Commun., vol. 61, no. 6, pp. 2350-2361, Jun. 2013.

[13] A. M. Tulino and S. Verdú, "Random matrix theory and wireless communications," Foundations and Trends in Communications and Information Theory, vol. 1, no. 1, pp. 1-182, Jun. 2004. 